\title{
Comparison of the efficacy of diclofenac and betamethasone following strabismus surgery
}

\author{
Mark Wright, Zahida Butt, Gawn McIlwaine, Brian Fleck
}

\begin{abstract}
Aims-To compare the relative antiinflammatory potency and safety of topical diclofenac-gentamicin with betamethasone-neomycin following strabismus surgery.

Methods-A single centre, single observer, prospective, randomised, and double masked clinical trial of 25 children undergoing bilateral symmetrical horizontal strabismus surgery was carried out. One eye received diclofenacgentamicin and the contralateral eye received betamethasone-neomycin; both treatments were instilled four times a day for 4 weeks postoperatively. Ocular inflammation was assessed at 1 and 4 weeks postoperatively, objectively by comparison with a photographic chart and subjectively by questionnaire.

Results-There was no statistically significant difference in the rate of resolution of the inflammatory response between each group at both visits.

Conclusion-Diclofenac appears to be as effective as betamethasone in controlling postoperative inflammation following strabismus surgery and may offer a safer alternative to the use of topical steroids. (Br F Ophthalmol 1997;81:299-301)
\end{abstract}

\section{Patients and methods}

Written informed consent was obtained from all parents of 25 children aged between 1 and 12 years of age undergoing primary bilateral symmetrical surgery for eso- or exodeviations. Exclusion criteria included previous ocular surgery, a history or signs of ocular inflammation, hypersensitivity to non-steroidal antiinflammatory drugs (NSAIDs) or any other component of the study drugs.

All surgery was performed by or under the direct supervision of one surgeon (GMcI). Conjunctival incisions were either limbal or over the muscle insertions, esodeviations were realigned by bilateral medial rectus recessions, exodeviations by lateral rectus recessions. The recessed muscles were reattached with $5 / 0$ Vicryl and the conjunctival incisions were closed with 8/0 Vicryl.

One eye was randomised to receive topical treatment with betamethasone sodium phosphate $0.1 \%$-neomycin sulphate $0.5 \%$ while the contralateral eye was treated with diclofenac sodium $0.1 \%$-gentamicin sulphate $0.3 \%$. Both treatments were instilled four times per day for 4 weeks postoperatively. All trial medications were produced and supplied by Ciba Vision
Ophthalmics and were packaged in identical bottles labelled right or left eye.

Patients were evaluated at 1 and 4 weeks postoperatively by one investigator (MW). The conjunctival inflammatory response was analysed objectively by comparing the degree of conjunctival injection over the sites of the muscle surgery against a series of colour photographs and allocating a 'conjunctival inflammatory score' for each eye. A score of 5 denoted a severe inflammatory response and a score of 0 denoted the absence of inflammation. Patient symptoms were analysed subjectively by parental questionnaire assessing ocular pain, conjunctival discharge, and drop tolerance. The severity of each symptom was graded on a scale of 0 (asymptomatic) to 3 (severely affected). All statistical analysis was performed using the Wilcoxon signed rank test.

\section{Results}

A total of 25 out of 29 children fulfilling the selection criteria were successfully recruited and follow up was complete. The mean age of the study population was 4.3 years with a range of 1-9 years, of the 25 patients, 17 were male and 21 had an esodeviation.

The mean conjunctival inflammatory score 1 week postoperatively for the diclofenacgentamicin group was 3.0 (SD 0.7) compared with a mean of $2.8(0.9)$ for the betamethasone-neomycin group (Fig 1 (upper)). There was no statistically significant difference between the two treatments, $p=$ 0.12 . The mean conjunctival inflammatory scores at 4 weeks postoperatively were $1.0(0.9)$ and $0.9(0.8)$ respectively for each group, with no statistically significant difference between treatments, $\mathrm{p}=0.255$ (Fig 1 (lower)).

Analysis of the results of the questionnaire demonstrated that strabismus surgery was well tolerated with all of the children having only mild or no discomfort 1 week postoperatively and every child being pain free after 4 weeks. One child had a mild conjunctival discharge (at visit 2 in the betamethasone-neomycin treated eye) which resolved spontaneously. Both treatments were well tolerated with parents reporting either no $(76 \%)$ or only mild $(24 \%)$ discomfort after instillation of the drops with no difference between treatment groups.

\section{PROTOCOL VIOLATIONS}

One patient inadvertently stopped the treatment 1 week early and a further two patients instilled the treatment less frequently than instructed, three times and twice daily respectively. The 


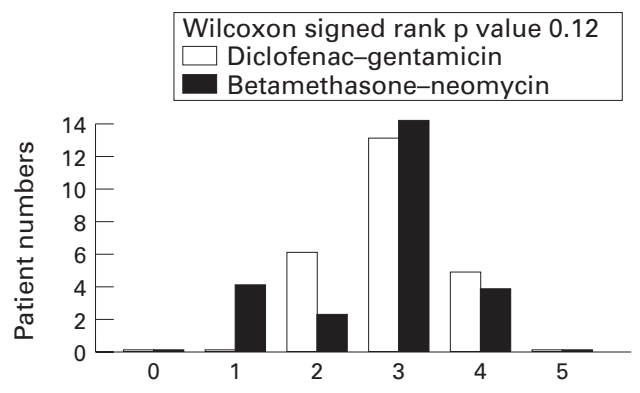

Conjunctival inflammatory scores

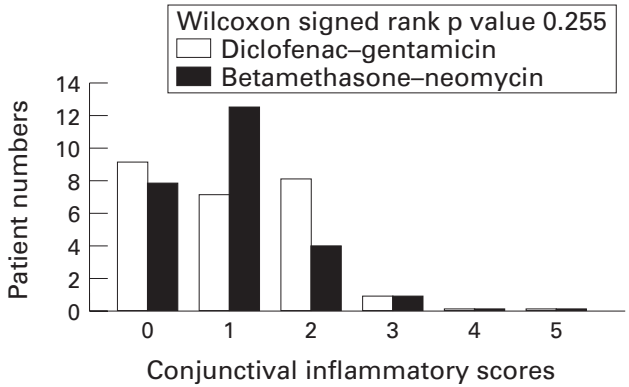

Figure 1 (Upper) Visit 1. Conjunctival inflammatory scores 1 week postoperatively. (Lower) Visit 2. The conjunctival inflammatory scores 4 weeks postoperatively.

data from these three patients were still included and analysed as the effects were symmetrical and should not bias the final result.

\section{ADVERSE EVENTS}

Two patients returned at 1 and 15 days respectively after completion of the trial with bilateral conjunctival injection. Both settled spontaneously within 2 days of presentation.

\section{Discussion}

Ideally, the two treatments studied would only have differed by the nature of the antiinflammatory; unfortunately no steroidgentamicin combination is commercially available in the UK (Ciba Vision Ophthalmics chose gentamicin in preference to neomycin because of its marginally superior antibacterial activity). Betamethasone-neomycin is the most commonly prescribed steroid-antibiotic combination and was chosen as the treatment with which to compare diclofenac-gentamicin. The primary objective of this clinical trial was to compare the relative anti-inflammatory potencies of the combination of diclofenacgentamicin and betamethasone-neomycin rather than their relative antibacterial efficacy.

The controversy regarding the merits of the routine use of topical antibiotics alone or in conjunction with an anti-inflammatory agent following strabismus surgery has continued for years. The results from some clinical trials have suggested that antibiotic treatment should be used routinely, ${ }^{1}$ others have concluded that treatment with anti-inflammatory agents such as sulphacetamide-prednisolone ${ }^{2}$ or oxyphenbutazone-choramphenicol ${ }^{3}$ was not beneficial. Sight threatening infectious complications following strabismus surgery are rare and include endophthalmitis (which is related to scleral perforation and has a reported incidence of one case per 3500-8000 operations $^{45}$ ) and orbital cellulitis. ${ }^{6}$ It would be extremely difficult because of the rarity of these events to prove that the incidence of these severe complications were affected by the routine prescription of topical antibiotics used alone or in combination with antiinflammatory agents.

The results of this prospective randomised clinical trial suggest that following strabismus surgery, diclofenac-gentamicin and betamethasone-neomycin are equally effective and well tolerated topical anti-inflammatory/antibacterial agents. The conjunctival inflammatory scores of the three patients instilling the treatment less frequently than instructed were consistently higher than the mean scores of both treatment groups at both 1 and 4 weeks postoperatively. It can be inferred that both treatments significantly reduced inflammation.

Many surgeons continue routinely to prescribe a topical antibiotic-steroid combination for up to 1 month postoperatively. Raised intraocular pressure (IOP), increased susceptibility to infections, in particular herpetic keratitis, and delayed wound healing are all recognised side effects of topical steroids. ${ }^{7}$ Ohji et $a l^{8}$ demonstrated that $82 \%$ of children under 10 years of age receiving topical dexamethasone $0.1 \%$ three times daily following strabismus surgery demonstrated significant elevations of IOP with half of the children having rises in IOP of greater than $15 \mathrm{~mm} \mathrm{Hg}$. NSAIDs possess none of these characteristics and in addition have intrinsic analgesic properties. ${ }^{9}$ The anti-inflammatory properties of topical diclofenac have compared favourably with those of steroids following cataract surgery ${ }^{1011}$ and excimer laser. ${ }^{12}$ Diclofenac directly modulates the cyclo-oxygenase pathway and indirectly modulates the lipoxygenase pathway reducing the production of prostaglandins and leukotrienes which are the main mediators of the inflammatory response. ${ }^{13}$ While there was no observable increase in the degree of local subconjunctival haemorrhage following the use of topical diclofenac, it has been reported that topical flurbiprofen may cause an increase in the bleeding tendency of ocular tissues in conjunction with surgery. ${ }^{14}$

In conclusion, diclofenac appears to be as effective as betamethasone in controlling postoperative inflammation following strabismus surgery. NSAIDs have potentially fewer serious ocular side effects than topical steroids, therefore we feel that diclofenac-gentamicin can be recommended as a valid alternative to steroidantibiotic combinations.

The authors have no proprietary interest in Ciba Vision Ophthalmics or their products.

1 Kearns PP, Cullen JF. Fucithalmic, chloramphenicol or no reatment after squint surgery in children. Acta Ophthalmol 1992;70:132-4.

2 Wortham E, Anandakrishnan I, Kraft SP, Smith D, Morin JD. Are antibiotic-steroid drops necessary following JD. Are antibiotic-steroid drops necessary following
strabismus surgery? A prospective, randomised, masked trial. F Pediatr Ophthalmol Surg 1990;27:205-7.

3 Hagan MC, Dinning WJ. Day case strabismus surgery without postoperative ocular medication. A masked ranout postoperative ocular medication
domised study. Eye 1987;1:581-4. 
4 Knobloch $\mathrm{R}$ and Lorenz A. Ueber ernste komplikationen nach schieloperationen. Klin Monatsbl Augenheilkd 1962;

5 Weinstein GS, Mondino BJ, Weinberg RJ, Biglan AW. Endophthalmitis in a paediatric population. Ann Ophthalmol 1979;6:935-43.

6 Von Noorden GK. Orbital cellulitis following extraocular muscle surgery. Am $\mathcal{f}$ Ophthalmol 1972;74:627-9.

7 Havener WH. Corticosteroid therapy. In: Ocular pharmacology. 4th ed. St Louis: CV Mosby, 1978:143-74.

8 Ohji M, Kinoshita S, Ohmi E, Kuwayama Y. Marked intraocular pressure response to installation of corticosteroids in children. Am 7 Ophthalmol 1991;112:450-4.

9 Kaidman GW. Diclofenac and its effect on corneal sensation. Arch Ophthalmol 1995;113:262.

10 Othenin-Girard P, Tritten J, Pittet N, Herbort C. Dexamethasone versus diclofenac sodium eyedrops to treat inflammation after cataract surgery. 7 Cataract Refract Surg 1994;20:9-12.

11 Kraft MC, Sanders DR, McGuigan L, Gold Rannan M. Inhibition of blood-aqueous humor barrier breakdown with diclofenac, a fluorophotometric study. Arch Ophthalmol 1990;108:380-3.

12 Sher NA, Frantz JM, Talley A, Parker P, Lane SS, Ostrov C, et al. Topical diclofenac in the treatment of ocular pain after excimer photorefractive keratectomy. Refract Corneal Surg 1993;9:425-36.

$13 \mathrm{Ku} \mathrm{EC,} \mathrm{Lee} \mathrm{W,} \mathrm{Kothari} \mathrm{HV,} \mathrm{Scholer} \mathrm{DW.} \mathrm{Effect} \mathrm{of}$ diclofenac sodium on the arachidonic acid cascade. Am $\mathcal{F}$ Med 1985;80:18-23.

14 Ferrari $M$. Use of topical non-steroidal anti-inflammatory drugs photorefractive keratectomy. F Refract Corneal Surg 1994;10(2 suppl): 87-9. 